\title{
Jovens Programadores: ensino de programação e robótica para alunos do ensino básico de Monte Carmelo-MG
}

\author{
Emmanuel F. A. Reis ${ }^{1}$, Phelipe R. Santos ${ }^{1}$, Bruna L. Dutra ${ }^{1}$, \\ Leandro N. Couto ${ }^{1}$, Daniele C. Oliveira ${ }^{1}$, Murillo G. Carneiro ${ }^{1}$ \\ ${ }^{1}$ Faculdade de Computação - Universidade Federal de Uberlândia (UFU) \\ Uberlândia, MG, Brasil \\ \{emmanureis, pheliperodovalho\}@gmail.com, \\ \{bruna.dutra, leandronc, danieleoliveira, mgcarneiro\}@ufu.br
}

\begin{abstract}
This paper describes the methodology developed in an extension program aimed at teaching computer programming and robotics to elementary and high school students in the city of Monte Carmelo-MG, the Jovens Programadores. To be specific, we analyze and discuss data obtained from the program in the first semester of 2018. Among the results obtained, it is worth highlighting the broad reach of the program, which has students from all schools in the city as well as the level of involvement of a good part of the students.
\end{abstract}

Resumo. Este artigo descreve a metodologia de trabalho desenvolvida em um programa de extensão voltado para o ensino de programação e robótica para alunos do ensino fundamental e médio da cidade de Monte Carmelo-MG, o Jovens Programadores. Especificamente, dados obtidos pelo programa no primeiro semestre de 2018 são analisados e discutidos de maneira quantitativa e qualitativa. Entre os resultados obtidos, merece destaque a representatividade do programa, que inclui alunos de todas as escolas da cidade, bem como o alto nível de envolvimento de boa parte dos alunos.

\section{Introdução}

Computadores são ferramentas cada vez mais pervasivas na sociedade, presentes em todas as áreas da vida moderna, de diversas formas. Por isso, compreender o funcionamento de tais ferramentas e saber programá-las para desenvolver soluções novas, próprias e personalizadas, são conhecimentos extremamente atuais e relevantes, embora dominados por uma fração relativamente pequena das pessoas que fazem uso dessas mesmas tecnologias. Assim, o ensino e aprendizado de programação e robótica é importante para todos. Douglas Rushkoff [Rushkoff 2010] propõe uma analogia entre as restrições de acesso à escrita e leitura, que um dia já foram saberes restritos a elites intelectuais e outras classes sociais determinadas, e o atual elitismo e restrição do conhecimento de computação, programação e eletrônica. Da mesma forma que a escrita e leitura se popularizaram entre pessoas de todas as classes sociais, assim deve ser com a programação, devido à influência fundamental e crescente dos computadores na vida de todos.

Segundo dados do IBGE de 2016, o Brasil somava 64,7\% da população, ou seja, 116 milhões de pessoas com acesso à computadores e à internet. A internet em particular é um palco importante de comunicação e influência, e o domínio da tecnologia é uma 
ferramenta de inclusão, o que levou a professora Clarisse de Souza ${ }^{1}$ a afirmar que "Tão importante quanto saber falar por si, para que ninguém fale por você, é saber programar por si, para não ser programado".

O ensino de programação e robótica desde a infância também tem sido aplicado com sucesso em diversos experimentos ao redor do mundo [Lye and Koh 2014]. Ainda podemos notar que a popularização da engenharia de software e a redução do custo dos equipamentos tem permitido o desenvolvimento de ferramentas de software e hardware acessíveis e voltadas ao ensino, sempre tendo em vista que a fluência digital transcende o simples papel de usuário, mas envolve também o papel de criador, projetista e adaptador [Resnick et al. 2009]. Além da inclusão e fluência digital que o ensino de programação e robótica promove, ele também serve de catalisador no aprendizado de demais disciplinas das Ciências Exatas, como matemática, física e lógica, envolvendo conhecimentos de tais tópicos em tarefas lúdicas e criativas de resolução de problemas. Dada a relevância do tema, a própria Sociedade Brasileira de Computação trabalha nos últimos anos em uma proposta para integrar o ensino de computação na Educação Básica² .

Este trabalho descreve a experiência relativa ao programa Jovens Programadores considerando o primeiro semestre de 2018 (2018/1). Especificamente, o programa envolve o ensino de programação e robótica a alunos de escolas públicas e privadas da rede de ensino básico da cidade de Monte Carmelo - MG, através de cursos gratuitos oferecidos no campus da Universidade Federal de Uberlândia. Tal experiência foi alicerçada no desenvolvimento de um currículo prático, que despertasse o interesse dos alunos, que pudesse ser ministrado por discentes da universidade, sob orientação de docentes, e que pudesse ser desenvolvido de forma eficaz em um semestre de curso.

O restante do trabalho é organizado como segue. A Seção 2 apresenta trabalhos relacionados que embasaram o planejamento do Jovens Programadores, a Seção 3 descreve a metodologia proposta, a Seção 4 apresenta os resultados obtidos e uma análise do mérito da ação realizada, e a Seção 5 apresenta considerações finais do trabalho.

\section{Trabalhos Relacionados}

A literatura tem evidenciado o potencial educativo da introdução do ensino de robótica e programação para alunos de ensino básico na capacitação para solução de problemas que envolvam criatividade e lógica [Lux et al. 2007, Benitti et al. 2009].

Para alcançar os alunos de ensino básico com o ensino de programação e robótica, busca-se por vezes abordagens adequadas à faixa etária. Por exemplo, [Costa et al. 2016] apresenta um trabalho realizado com alunos do ensino fundamental da rede pública para o ensino de lógica e programação. O curso foi distribuído por módulos, introduzindo os conceitos básicos de lógica em atividades lúdicas. Os alunos foram divididos em grupos o que instigou a competição entre eles contribuindo para prepará-los para a Olimpíada Brasileira de Informática.

Outro importante trabalho relativo ao ensino de programação é apresentado em [Garcia et al. 2008], onde os autores utilizaram uma abordagem orientada a problemas. Inicialmente, foram introduzidos conceitos básicos de programação em Português

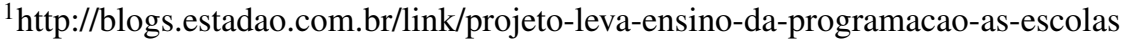

${ }^{2}$ http://www.sbc.org.br/files/ComputacaoEducacaoBasica-versaofinal-julho2017.pdf
} 
VII Congresso Brasileiro de Informática na Educação (CBIE 2018)

Anais dos Workshops do VII Congresso Brasileiro de Informática na Educação (WCBIE 2018)

estruturado. Contudo, após constatarem que um ambiente de programação poderia motivar os alunos, os autores optaram pela linguagem C. De acordo com a evolução dos alunos outros conceitos e problemas mais complexos puderam ser explorados, fundamentando a conclusão dos autores de que o estudo de algoritmos pode ser compreendido independente da idade e se trabalhado desde cedo pode ajudar a diminuir a evasão dos cursos de Tecnologia da Informação.

Em [Oliveira et al. 2014], os autores relatam a experiência do ensino de lógica de programação para alunos do $9^{\circ}$ ano do fundamental utilizando a ferramenta Scratch. Com a introdução dos conceitos básicos por meio de atividades de computação desplugada, incentivando a criatividade com a construção de histórias interativas trabalhando matemática e computação, foi possível desenvolver jogos simples ao final do curso. Com liberdade para explorar seus conhecimentos, os alunos foram instigados a realizarem suas próprias descobertas e desenvolverem no Scratch de acordo com seus interesses.

Em [Marques et al. 2011] foi ministrada uma oficina de programação básica no ensino médio, em que utilizou-se a linguagem Python para a criação de jogos. Nas aulas, os alunos utilizaram um programa desenvolvido pelos instrutores para ajudar na compreensão sobre o funcionamento do computador, além de introduzir conceitos sobre lógica de programação e estrutura de dados. Apesar de o projeto ter tido duração de apenas quatro dias, os alunos após a oficina demonstraram interesse por cursos superiores em computação.

Além do ensino de programação de computadores, o Jovens Programadores também oferece cursos de robótica. Uma das plataformas mais populares para o ensino de robótica na escola é o LEGO Mindstorms [Hussain et al. 2006] [Rollins 2014]. Uma das vantagens observadas na plataforma Mindstorms está a facilidade na montagem e personalização de robôs, além de interface simplificada para gravação de programas no microcontrolador embarcado NXT e interface de programação visual e intuitiva, baseada em blocos e fluxo.

Por outro lado, a plataforma LEGO Mindstorms é uma plataforma de software e hardware proprietários e de custo elevado, o que a torna de difícil acesso para muitas escolas brasileiras. A programação dos robôs usando o microcontrolador NXT também abstrai ou obscurece muitos aspectos eletrônicos do robô, o que consideramos desejável no caso de ensino de lógica de programação, mas uma característica negativa no caso do ensino de fundamentos de eletrônica e robótica.

O trabalho de Kafai et al. [Kafai et al. 2014], por exemplo, corrobora a ideia de que uma abordagem mais manual, com o uso de sistemas como Arduino e materiais de fácil acesso, se apresentada através de problemas cuidadosamente formulados, pode despertar grande curiosidade e engajamento dos alunos. Balogh [Balogh 2010] também aponta como fatores positivos para o uso educativo da plataforma Arduino o uso da difundida linguagem de programação $\mathrm{C} / \mathrm{C}++$ e a popularidade da plataforma, características que garantem uma vasta e ativa comunidade online, o que facilita a obtenção de ajuda, materiais e exemplos, incentivando a pesquisa.

O trabalho de Júnior et al. [Junior et al. 2017], enfatiza a importância da robótica no ensino médio auxiliando os alunos na disciplina de matemática. O tópico escolhido para a realização das atividades foi funções de $1^{o}$ grau. Inicialmente foi introduzido 
o conteúdo de funções e seguidamente os alunos utilizaram os robôs aplicando os conhecimentos teóricos para resolução das atividades. O resultado foi satisfatório, visto que os alunos ficaram mais interessados nas soluções dos problemas, tornando o conteúdo mais interessante e obtendo uma melhor compreensão sobre o tema.

\section{Metodologia}

As aulas dos cursos oferecidos pelo Jovens Programadores foram realizadas na Universidade Federal de Uberlândia, usando a infra-estrutura universitária de laboratórios e equipamentos. Essa decisão baseou-se no aspecto positivo da aproximação do aluno da realidade universitária, cumprindo o propósito universitário de extensão, e na reunião de alunos de escolas diferentes.

Foram oferecidos: ensino de programação de computadores e programação com robótica, cada curso com três níveis: Iniciante, Intermediário e Avançado. Com proposta de duração de 1 semestre completo, sendo 2 aulas semanais de 1h30min, ministradas por alunos extensionistas da universidade, havendo, em cada turma, um aluno bolsista acompanhado por um voluntário. Isso para que os universitários pudessem se apoiar ao ministrar as aulas teóricas e também no atendimento aos alunos durante as práticas.

Cada turma contempla até 20 vagas, destinadas a alunos do $8^{\circ}$ e $9^{\circ}$ ano do ensino fundamental e de todo o ensino médio, sendo oferecidas através de ampla divulgação (visitas nas escolas, rádio e panfletos) para todas as escolas públicas e privadas da cidade de Monte Carmelo - MG que atendem a faixa etária desejada. O número de turmas foi definido de acordo com a demanda dos alunos e o número de bolsistas do programa, sendo normalmente de 2 a 3 turmas por bolsista. O período das turmas (manhã, tarde ou noite), as modalidades e os níveis oferecidos a cada semestre também foram flexibilizados e definidos de acordo com a demanda dos inscritos.

A inscrição dos alunos interessados em participar do programa é realizada por um sistema de inscrição próprio, capaz de gerar relatórios importantes sobre as demandas. Assim, buscou-se criar um canal de comunicação online entre a universidade e os alunos, pais ou responsáveis. Para preenchimento das vagas, propôs-se que fosse usada a ordem de inscrição.

Os instrumentos utilizados para acompanhamento e avaliação do andamento do programa são: lista de presença, resultado ou pontuação obtidas em atividades específicas e questionário de avaliação por parte dos alunos.

No semestre contemplado pelo artigo (2018/1), foram oferecidos cursos de programação de computadores e robótica, ambos no nível Iniciante. O conteúdo abordado e metodologia de curso propostos foram:

- Programação de computadores: Fundamentos de lógica, introdução a algoritmos e linguagem de programação, constantes e variáveis, estruturas de controle e repetição, e resolução de problemas. A abordagem compreende inicialmente uma introdução com a linguagem de programação Portugol para a melhor compreensão dos alunos, e posteriormente é adotada a linguagem $\mathrm{C} / \mathrm{C}++$. Essa linguagem foi escolhida devido a uma parceria com uma empresa apoiadora do programa, a qual oferece suporte para competições de programação nesta linguagem. Para 
VII Congresso Brasileiro de Informática na Educação (CBIE 2018)

Anais dos Workshops do VII Congresso Brasileiro de Informática na Educação (WCBIE 2018)

o desenvolvimento dos alunos, também é utilizada a ferramenta de resolução de problemas URI ONLINE JUDGE ${ }^{2}$ e maratonas de programação com codepit $^{3}$.

- Robótica: Fundamentos de lógica, introdução a algoritmos e linguagem de programação, constantes e variáveis, estruturas de controle e repetição, introdução à plataforma Arduino e suas funções básicas, introdução a sensores e atuadores, introdução a eletrônica, desenvolvimento de aplicações e soluções com o kit de robótica. No caso da robótica, o objetivo foi utilizar uma plataforma inspirada no bem-sucedido LEGO Mindstorms, mas que também fosse de baixo custo, preferivelmente de software e hardware abertos, que oferecesse um ciclo ágil de desenvolvimento e teste e que permitisse aos alunos um contato realista com a robótica e a eletrônica. Para esse fim, elaboramos um kit de robótica baseado na plataforma Arduino, incluindo diversos sensores e atuadores de fácil manuseio e montagem. O kit escolhido obteve um retorno positivo dos alunos participantes em termos de manutenção do interesse e aprendizagem [Sousa et al. 2016]. A Figura 1 apresenta um dos robôs usado no programa, montado juntamente com os estudantes.

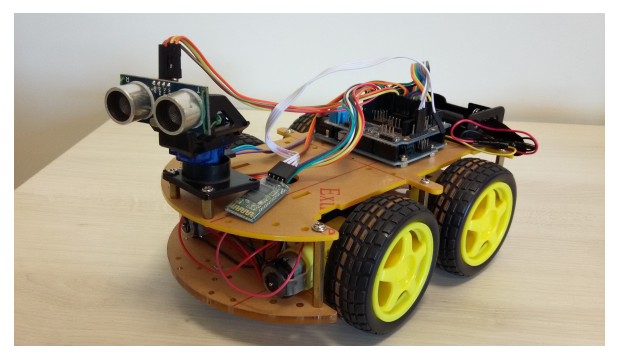

Figura 1. Robô montado com parte do kit de robótica usado nas aulas do Jovens Programadores.

\section{Análise e Discussão}

A análise e discussão apresentadas nesta seção são divididas em três partes: perfil dos candidatos inscritos, ensino de programação e robótica e avaliação por parte dos alunos.

\subsection{Perfil dos candidatos inscritos}

Para o semestre 2018/1, o programa contou com 188 inscrições. A Figura 2a apresenta o grau de escolaridade dos alunos. As inscrições foram abertas a alunos do $8^{\circ}$ e $9^{\circ}$ ano do ensino fundamental e de todo o ensino médio. Como pode ser observado, a maioria dos alunos são do $8^{\circ}$ ano $(\approx 40 \%)$, seguidos por alunos do $1^{\circ}$ ano do ensino médio e do $9^{\circ}$ ano do fundamental $(\approx 18 \%)$. Por outro lado, esse resultado sugere que boa parte dos alunos do $2^{\circ}$ e $3^{\circ}$ ano do ensino médio preferiram não participar do programa por estarem envolvidos com cursos preparatórios para vestibular. De fato, em edições anteriores do programa os alunos desses dois anos alegaram incompatibilidade de horário com cursos prepatórios para vestibular, o que levou muitos a desistirem de continuar participando do programa.

\footnotetext{
${ }^{2}$ https://www.urionlinejudge.com.br/

${ }^{3}$ https://www.codepit.io
} 


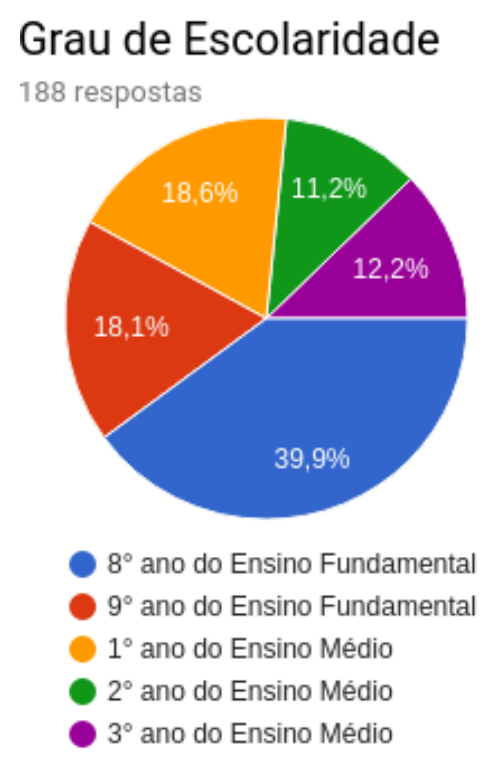

(a) Grau de escolaridade

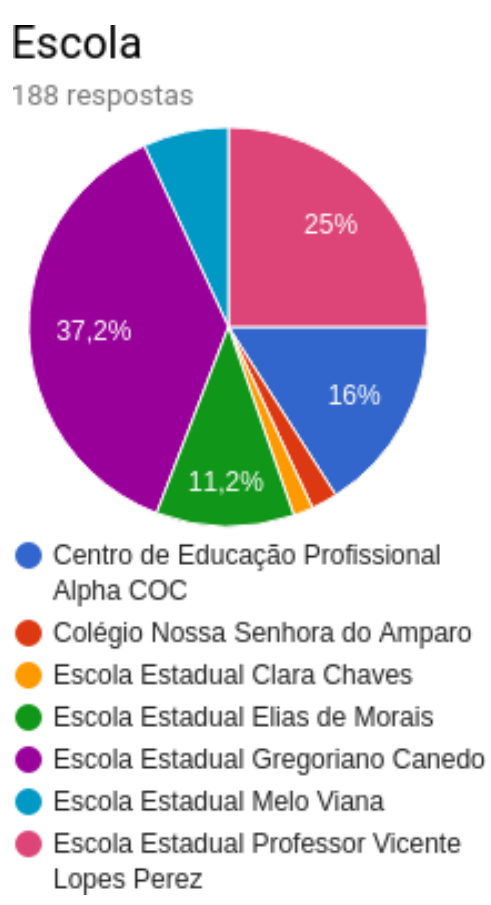

(b) Escolas

Figura 2. Perfil dos alunos inscritos no Jovens Programadores em 2018/1: (a) grau de escolaridade; (b) escolas onde estudavam.

Outra análise interessante sobre o perfil dos alunos do programa é relacionada à escola onde tais alunos estudam. De acordo com a Figura $2 b$, a maioria dos participantes são alunos da Escola Estadual Gregoriano Canedo $(\approx 37 \%)$, seguida pela Escola Estadual Professor Vicente Lopes Perez (25\%) e pelo Centro de Educação Profissional Alpha COC (16\%). É interessante observar que há alunos inscritos de todas as escolas de Monte Carmelo-MG, dentre as enquadradas no público alvo do programa.

Em relação à participação dos alunos inscritos no Jovens Programadores em semestres anteriores do programa, observamos que quase $87 \%$ dos alunos inscritos estavam participando do Jovens Programadores pela primeira vez, o que sugere algum resultado positivo na divulgação do programa. Em relação ao baixo número de alunos com participações anteriores, vale ressaltar que foram oferecidas apenas cursos de nível iniciante em 2018/1, o que pode ter impactado no menor número de inscritos com participação anterior no programa.

A última análise relacionada ao perfil dos alunos inscritos no Jovens Programadores refere-se ao curso de interesse deles. Segundo os dados coletados dos candidatos inscritos, pouco mais da metade deles $(\approx 55 \%)$ optaram pelo curso de programação, enquanto $\approx 45 \%$ pelo curso de robótica, o que revela uma demanda considerável por parte de ambos os cursos.

\subsection{Ensino de programação e robótica}

Ambos os cursos apresentaram resultados satisfatórios. Apesar de algumas desistências, as estratégias adotadas em cada curso foram capazes de auxiliar os alunos no aprendizado bem como mantê-los motivados durante as aulas. 
Para o curso de programação de computadores, foi adotada uma estratégia de ensino voltada à gameficação, com desafios e competições para os alunos. Nesse sentido, em todas as aulas foram apresentados aos alunos conceitos e desafios a serem resolvidos usando tais conceitos. Em relação às competições, foram realizadas maratonas de programação entre os alunos, inclusive premiando aqueles com melhor colocação. Segundo relato do bolsista que ministrou os cursos de programação, de início era perceptível a dificuldade de desenvolvimento de uma resolução para um problema proposto, algo que no final do curso já parecia muito mais simples depois do incentivo da pesquisa e resolução do problema por meio de recompensas ou prêmios.

Para o curso de robótica, a estratégia de ensino adotada foi baseada no kit de robótica, o qual é utilizado para prover uma aplicação prática relacionada a cada conceito aprendido. Dessa forma, a partir de montagens simples, como um semáforo por exemplo, os alunos assimilavam conceitos básicos para as tarefas seguintes, cujo grau de dificuldade aumentava gradualmente entre uma tarefa e outra. Segundo relato do bolsista que ministrou os cursos de robótica, boa parte dos alunos gostaram de aprender sobre robótica, especialmente das atividades práticas.

\subsection{Avaliação dos alunos}

No início e no término de cada semestre, a coordenação do programa se reúne com os alunos participantes para um diálogo acerca de expectativas e resultados, respectivamente. Entre as avaliações negativas, houve uma reclamação em relação à velocidade em que o conteúdo da aula é ministrado e outra relacionada à falta de espaço para trabalhar no computador e no kit de robótica. Entre as avaliações positivas destacam-se o nível de satisfação dos alunos, que se declararam muito satisfeitos com o programa, bem como a motivação para a continuidade dos cursos de programação e robótica no nível seguinte (intermediário).

Além da avaliação através de diálogo, os alunos também receberam por email um questionário avaliativo online. O questionário foi enviado para 57 alunos, dos quais 12 responderam. Por razões de espaço, as perguntas não são apresentadas no artigo, embora possam ser acessadas online em https://goo.gl/forms/ zmVqgy 8MjfySJoiI2.

Em relação à divulgação do JP na cidade de Monte Carmelo, é preciso melhorar: apenas $25 \%$ dos alunos concordam que o programa é bem divulgado na cidade; $58,3 \%$ concordam parcialmente; $8,3 \%$ discordam; e 8,3\% não possuem opinião. Contudo, a divulgação tem sido efetiva através das visitas nas escolas: $91,7 \%$ dos alunos souberam sobre o Jovens Programadores dessa forma; enquanto 8,3\% através de amigos ou terceiros.

Em relação ao conhecimento adquirido sobre computadores e programação durante o curso, os resultados são exibidos na Tabela 1. Na tabela é possível identificar um avanço notável no desenvolvimento dos alunos participantes do programa, especialmente em relação ao aprendizado dos conceitos de programação ensinados no curso.

Em relação às dificuldades encontradas pelos alunos nos cursos de programação, a maior delas é a interpretação de problemas (apontada por 5/8 alunos). A linguagem de programação (3/8), a matemática (2/8) e a explicação do professor (2/8) também foram consideradas. Ainda, 2/8 dos alunos apontaram não terem tido dificuldade alguma. Nos 
VII Congresso Brasileiro de Informática na Educação (CBIE 2018)

Anais dos Workshops do VII Congresso Brasileiro de Informática na Educação (WCBIE 2018)

\begin{tabular}{lccccc}
\hline \multirow{2}{*}{$\begin{array}{l}\text { Conhecimento } \\
\text { Avaliação }\end{array}$} & \multicolumn{2}{c}{ Sobre computadores } & & \multicolumn{2}{c}{ Sobre programação } \\
\cline { 2 - 3 } \cline { 6 - 6 } \cline { 5 - 6 } Muito Bom & Antes & Depois & & Antes & Depois \\
Bom & $8,3 \%$ & $16,7 \%$ & & $8,3 \%$ \\
Razoável & $25,0 \%$ & $\mathbf{6 6 , 7 \%}$ & & 0 & $\mathbf{6 6 , 7 \%}$ \\
Ruim & $\mathbf{5 0 , 0 \%}$ & $16,7 \%$ & & $16,7 \%$ & $25,0 \%$ \\
Muito Ruim & $16,7 \%$ & 0 & & $\mathbf{5 8 , 3 \%}$ & 0 \\
\hline
\end{tabular}

Tabela 1. Avaliação dos alunos sobre os conhecimentos sobre computadores e programação adquiridos durante o curso.

cursos de robótica, as dificuldades foram: uso dos componentes eletrônicos (1/5), falta de espaço para montagem e configuração dos robôs (1/5) e a linguagem de programação (1/5); 2/5 dos alunos informaram não terem tido dificuldade alguma.

Outra opção considerada por $25 \%$ dos alunos foi a quantidade insuficiente de aulas. De fato, 2018/1 foi um semestre atípico nesse sentido, pois houveram apenas 10 semanas de aula devido à ausência de recursos financeiros para contratação de bolsistas no início do semestre. Em um semestre normal, a quantidade de aulas são 14 semanas, sendo 4 horas-aula por semana.

O questionário também avaliou se houve contribuição do uso de competições de programação e de robôs para o ensino de programação, se a expectativa dos alunos sobre o curso foi cumprida e se houve melhoria do rendimento escolar. Basicamente, foi feita uma afirmação positiva sobre cada um destes itens e os alunos deveriam responder se concordavam, concordavam parcialmente, discordavam ou se não tinham opinião à respeito. Um sumário das respostas para cada item é apresentado na Tabela 2, a qual evidencia que a maior parte dos alunos concordam que a metodologia e ferramentas empregadas no programa refletem positivamente no seu aprendizado. Mais do que isso, muito desse aprendizado também contribuiu para um melhor desempenho escolar, evidenciando a importância desse tipo de iniciativa para a formação básica dos alunos.

\begin{tabular}{lcccc}
\hline Avaliação & Competições & Robôs & Expectativa & Rend. Escola \\
\hline Concordo & $\mathbf{5 / 8}$ & $\mathbf{5 / 5}$ & $\mathbf{5 8 , 3 \%}$ & $\mathbf{6 6 , 7 \%}$ \\
Concordo Parcial. & $2 / 8$ & 0 & $33,3 \%$ & $16,7 \%$ \\
Discordo & $1 / 8$ & 0 & $8,3 \%$ & 0 \\
Sem Opinião & 0 & 0 & 0 & $16,7 \%$ \\
\hline
\end{tabular}

Tabela 2. Avaliação do aprendizado dos alunos em termos da contribuição das competições de programação e do uso de robôs no aprendizado de programação, do cumprimento da expectativa dos alunos sobre o programa e da contribuição do programa sobre o rendimento escolar deles.

Por fim, o questionário também deixa um espaço aberto para que os alunos façam comentários e opinem sobre aspectos positivos e negativos do programa. Recebemos 6 comentários, todos eles positivos. A Tabela 3 apresenta alguns dos comentários enviados pelos alunos, os quais reforçam as discussões e análises realizadas nesta seção. 
VII Congresso Brasileiro de Informática na Educação (CBIE 2018)

Anais dos Workshops do VII Congresso Brasileiro de Informática na Educação (WCBIE 2018)

\section{Comentários dos alunos participantes do programa}

"Gosto de participar das aulas. Toda aula realizo problemas diversos que ampliam meu aprendizado a respeito de programação."

"É muito bom estar participando de um cursos em ambientes que antes não conhecia, que nos apresenta outra visão sobre os estudos, com isso conhecer um pouco do curso de Sistemas de Informação da UFU."

"foi muito bom,pelo o fato de melhorias nas matérias da escola."

"Ótima iniciativa da universidade. Sempre me interessei por programação, mas nunca tive muitas oportunidades. Agradeço aos professores e alunos que se voluntariaram para nos ensinar e nos forneceram essa grande oportunidade."

Tabela 3. Comentários enviados pelos alunos participantes do programa.

\section{Considerações Finais}

O programa de extensão Jovens Programadores tem por objetivo estimular e desenvolver o estudo de programação e robótica, fomentando o raciocínio lógico e matemático, o pensamento crítico, o encadeamento de ideias e o conhecimento tecnológico dos jovens do ensino básico de Monte Carmelo-MG. O trabalho de [Bordini et al. 2016] apresenta que este tipo de iniciativa vem crescendo cada vez mais no Brasil, desenvolvendo o pensamento computacional no ensino básico através de diferentes tipos de estratégias. Dentre as estratégias mencionadas pelos autores, duas delas são utilizadas no programa Jovens Programadores para atrair e desenvolver o interessse dos jovens pela área da computação, o ensino através de Algoritmos e Programação, e através da Robótica.

Dentre os aspectos positivos do JP, destaca-se o bom engajamento de jovens estudantes de escolas públicas, que nunca haviam tido a oportunidade de estudar aulas práticas em laboratório de computação e robótica, e visível desenvolvimento dos alunos que completaram o curso em relação ao ponto em que entraram. Foi possível aferir, também, a elevada satisfação dos alunos participantes do programa, os quais se mostraram muito satisfeitos com os cursos e a qualidade do aprendizado. Observamos a necessidade, porém, de elaborar estratégias para conter a evasão de alunos durante o semestre, com frequência motivada por dificuldades de horário e/ou locomoção, bem como ampliar a divulgação do programa na cidade. É digno de nota que, motivados pelas ações do programa, alguns alunos demonstraram interesse em atuar na área de computação.

\section{Referências}

Balogh, R. (2010). Educational robotic platform based on arduino. In Proceedings of the 1st international conference on Robotics in Education, RiE2010. FEI STU, Slovakia, pages 119-122.

Benitti, F. B. V., Vahldick, A., Urban, D. L., Krueger, M. L., and Halma, A. (2009). Experimentação com robótica educativa no ensino médio: ambiente, atividades e resultados. In Workshop de Informática na Escola, volume 1, pages 1811-1820. 
VII Congresso Brasileiro de Informática na Educação (CBIE 2018)

Anais dos Workshops do VII Congresso Brasileiro de Informática na Educação (WCBIE 2018)

Bordini, A., Avila, C. M. O., Weisshahn, Y., da Cunha, M. M., da Costa Cavalheiro, S. A., Foss, L., Aguiar, M. S., and Reiser, R. H. S. (2016). Computaçao na educaçao básica no brasil: o estado da arte. Revista de Informática Teórica e Aplicada, 23(2):210-238.

Costa, T., Cristiano, F., Martins, D., and da Silva, W. (2016). A importância da computação para alunos do ensino fundamental: ações, possibilidades e benefícios. In Workshop de Informática na Escola, volume 22, pages 593-601.

Garcia, R. E., Correia, R. C. M., and Shimabukuro, M. H. (2008). Ensino de lógica de programação e estruturas de dados para alunos do ensino médio. In Workshop sobre Educação em Computação, pages 246-249.

Hussain, S., Lindh, J., and Shukur, G. (2006). The effect of lego training on pupils' school performance in mathematics, problem solving ability and attitude: Swedish data. Educational Technology \& Society, 9(3):182-194.

Junior, J. D., Coelho, L. S., and Santos (2017). Roboótica nas aulas de matemática do ensino médio: uma proposta educacional e de baixo custo. Experiências em Ensino de Ciências, 12(5):82-104.

Kafai, Y. B., Lee, E., Searle, K., Fields, D., Kaplan, E., and Lui, D. (2014). A craftsoriented approach to computing in high school: Introducing computational concepts, practices, and perspectives with electronic textiles. ACM Transactions on Computing Education, 14(1):1-20.

Lux, B., Haetinger, W., Engelmann, E. H., Horn, F., and da Cruz, M. E. J. K. (2007). Formação prática do licenciando em computação para trabalho com robótica educativa. In Simpósio Brasileiro de Informática na Educação, volume 1, pages 340-349.

Lye, S. Y. and Koh, J. H. L. (2014). Review on teaching and learning of computational thinking through programming: What is next for k-12? Computers in Human Behavior, 41:51-61.

Marques, D. L., Costa, L. F. S., de Azevedo Silva, M. A., and Rebouças, A. D. D. S. (2011). Atraindo alunos do ensino médio para a computação: Uma experiência prática de introdução à programação utilizando jogos e python. In Workshop de Informática na Escola, volume 1, pages 1138-1147.

Oliveira, M. L. S., Souza, A. A., Ferreira, A., and Barbosa, E. F. S. B. (2014). Ensino de lógica de programação no ensino fundamental utilizando o scratch: um relato de experiência. In Workshop sobre Educação em Computação, pages 1493-1502.

Resnick, M., Maloney, J., Monroy-Hernández, A., Rusk, N., Eastmond, E., Brennan, K., Millner, A., Rosenbaum, E., Silver, J., Silverman, B., et al. (2009). Scratch: programming for all. Communications of the ACM, 52(11):60-67.

Rollins, M. (2014). Beginning Lego Mindstorms Ev3. Apress.

Rushkoff, D. (2010). Program or be programmed: Ten commands for a digital age. Or Books.

Sousa, L., Costa, D., Martinez, A. C., Ribeiro, T., Couto, L., and Souza, J. (2016). Ensino de programação em robótica móvel no ensino fundamental e médio. In Anais dos Workshops do Congresso Brasileiro de Informática na Educação, volume 5, pages 399-402. 